\title{
artigo
}

Rosin, B.E.; Machado, F.R.A.; Rosa, S.M.; Paszcuk, A.M.; Karstens, L.F.; Pinto, L.H.;

Influência dos fatores "sobrepeso" e "sedentarismo" no aparecimento precoce de diabetes em mulheres com SOP

\section{Influência dos fatores "sobrepeso" e "sedentarismo" no aparecimento precoce de diabetes em mulheres com SOP}

\author{
Influence of the factors "overweight" and "sedentarism" in the early appearance of diabetes in women with PCOS \\ Influencia de los factores "sobrepeso" y "sedentarismo" en la aparición temprana de diabetes en mujeres con SOP
}

\begin{abstract}
RESUMO
Objetivo: Conhecer a influência dos fatores de riscos modificáveis "sobrepeso" e "sedentarismo" no aparecimento precoce de diabetes melitus tipo 2 (DM 2) em mulheres com Síndrome do Ovário Policístico (SOP). Método: revisão da literatura com busca dos uni termos no esquema boolanao: (síndrome dos ovários policísticos AND diabetes mellitus tipo 2) OR (estilo de vida, obesidade e comportamento sedentário), nos sites PUBME e SciELO. Resultados: Foram encontrados 3611 artigos no PUBMED e 1713 no SciELO; no qual - após triagem pelos pesquisadores - encontrou-se 16 artigos que atendiam os critérios estabelecidos. Etnia trata-se de um fator que predispõe a risco, bem como tempo gasto com televisão e celulares, que elevam risco de obesidade em até 23\% e de DM 2 em mulheres com SOP em até 14\%. Conclusão: Necessidade de ações para as mulheres com SOP para evitar DM2 precoce, incluindo ações em planos municipais de saúde, com ênfase na atenção primária.
\end{abstract}

DESCRITORES: Síndrome dos Ovários Policísticos; Diabetes Mellitus Tipo 2; Estilo de Vida, Obesidade, Comportamento Sedentário.

\section{ABSTRACT}

Objective: To know the influence of modifiable risk factors "overweight" and "sedentary lifestyle" in the early onset of type 2 diabetes mellitus (DM 2) in women with Polycystic Ovary Syndrome (PCOS). Method: literature review with search for the terms in the boolean scheme: (polycystic ovary syndrome AND type 2 diabetes mellitus) OR (lifestyle, obesity and sedentary behavior), on the PUBME and SciELO websites. Results: 3611 articles were found in PUBMED and 1713 in SciELO; in which - after screening by the researchers - 16 articles were found that met the established criteria. Ethnicity is a factor that predisposes to risk, as well as time spent on television and cell phones, which increase the risk of obesity by up to $23 \%$ and DM 2 in women with PCOS by up to $14 \%$. Conclusion: Need for actions for women with PCOS to prevent early DM2, including actions in municipal health plans, with an emphasis on primary care.

DESCRIPTORS: Polycystic Ovary Syndrome; Type 2 Diabetes Mellitus; Lifestyle, Obesity, Sedentary Behavior.

\section{RESUMEN}

Objetivo: Conocer la influencia de factores de riesgo modificables "sobrepeso" y "estilo de vida sedentario" en la aparición precoz de la diabetes mellitus tipo 2 (DM 2) en mujeres con Síndrome de Ovario Poliquístico (SOP). Método: revisión de la literatura con búsqueda de los términos en el esquema booleano: (síndrome de ovario poliquístico Y diabetes mellitus tipo 2) 0 (estilo de vida, obesidad y comportamiento sedentario), en los sitios web PUBME y SciELO. Resultados: se encontraron 3611 artículos en PUBMED y 1713 en SciELO; en el que, tras un cribado por parte de los investigadores, se encontraron 16 artículos que cumplían con los criterios establecidos. La etnia es un factor que predispone al riesgo, así como el tiempo dedicado a la televisión y al celular, que aumentan el riesgo de obesidad hasta en un 23\% y de DM 2 en mujeres con SOP hasta en un 14\%. Conclusión: Necesidad de acciones para mujeres con SOP para prevenir la DM2 temprana, incluyendo acciones en los planes de salud municipales, con énfasis en la atención primaria.

DESCRIPTORES: Síndrome de Ovario Poliquístico; Diabetes Mellitus Tipo 2; Estilo de Vida, Obesidad, Comportamiento Sedentario.

RECEBIDO EM: 02/11/2020 APROVADO EM: 23/11/2020

\section{Bianca Elicker Rosin}

Graduando em Medicina, Universidade da Região de Joinville, UNIVILLE.

ORCID: 0000-0002-7174-5814 
Felipe Reinert Avilla Machado

Graduando em Medicina, Universidade da Região de Joinville, UNIVILLE.

ORCID: 0000-0002-3221-0438

\section{Sabrina Martina da Rosa}

Graduanda em Enfermagem, Universidade da Região de Joinville, UNIVILLE.

ORCID: 0000-0002-3852-1202

\section{Aline Mirian Paszcuk}

Graduanda em Enfermagem, Universidade da Região de Joinville, UNIVILLE.

ORCID: 0000-0002-6943-0688

\section{Luciana Ferreira Karstens}

Professora Adjunta, Departamento de Enfermagem, Universidade da Região de Joinville, UNIVILLE.

ORCID: 0000-0001-9843-3047

\section{Luciano Henrique Pinto}

Professor Adjunto, DepartamentoS de Medicina, Enfermagem e Farmácia, Universidade da Região de Joinville, UNIVILLE, Coordenador do Projeto Integrado ECOSAM.

ORCID: 0000-0003-0250-7502

\section{INTRODUÇÃO}

A Síndrome dos Ovários Policísticos (SOP) é uma doença multifatorial que se caracteriza por alterações hiperandrogênicas e reprodutivas. Sua etiologia é complexa, com influência da predisposição genética e de fatores ambientais. As principais manifestações clínicas incluem acne, hirsutismo, alopecia, alterações menstruais e infertilidade ${ }^{1}$.

Esta síndrome é uma das desordens endocrinológicas mais frequentes em mulheres na idade reprodutiva, com prevalência de 6 a $10 \%$. Estima-se que, no mundo todo, 105 milhões de mulheres entre 15 e 49 anos de idade (sendo 4 milhões americanas) apresentem a SOP, a qual é responsável por 72 a $82 \%$ das causas de hiperandrogenismo ${ }^{1}$. O grupo de Pesquisa ECOSAM da Universidade da Região de Joinville vem realizando estudos sobre a possibilidade do aumento desta prevalência devido a presença de interferentes endócrinos em recursos hídricos e que podem contribuir para surgimentos de casos de SOP. Atualmente, usa-se os critérios de diagnóstico do The Rotterdam Concensus que, em 2003, definiu que fossem consideradas com SOP aquelas pacientes em que se encontrassem com as características: oligo-ovulação e/ou anovulação, hiperandrogenismo clínico ou bioquímico e a presença de ovários policísticos ${ }^{2}$.
A Síndrome dos

Ovários Policísticos

(SOP) é uma doença

multifatorial que

se caracteriza

por alteraçôes

hiperandrogênicas

e reprodutivas. Sua

etiologia é complexa,

com influência

da predisposição

genética e de fatores

ambientais.
Na maioria dos casos o tratamento da síndrome do ovário policístico visa o controle dos sintomas de hiperandrogenismo, restauração dos ciclos ovulatórios, correção da síndrome metabólica e proteção endometrial ${ }^{3}$. Além disso, o tratamento visa diminuir os aspectos estéticos que tendem a afetar o bem-estar da mulher, tais quais o hirsutismo, acne, seborreia, alopecia encontram-se como os principais. Dentre as principais medidas tomadas pela mulher portadora da síndrome alterações no estilo de vida estão os tratamentos hormonais, tratamento estético e o uso de sensibilizadores de insulina são as mais indicadas. Assim, o tratamento da patogenia tem como objetivo diminuir os sintomas e melhorar o bem estar da mulher portadora da síndrome ${ }^{4}$.

Considerando que a síndrome detém relação tanto à resistência insulínica quanto ao perfil metabólico individual, se apresenta então como forte fator predisponente para desenvolvimento de doenças. Tal condição pode diminuir a sobrevida das mulheres, e prejudicar sua qualidade de vida como um todo. A resistência insulínica pode contribuir para o desenvolvimento da diabetes mellitus do tipo 2 (DM 2); que pode ser precocemente desenvolvida se houver associação com alimentação inadequada para pré-diabéticos, sobrepeso e sedenta- 


\section{artigo}

Rosin, B.E.; Machado, F.R.A.; Rosa, S.M.; Paszcuk, A.M.; Karstens, L.F.; Pinto, L.H.;

Influência dos fatores "sobrepeso" e "sedentarismo" no aparecimento precoce de diabetes em mulheres com SOP

rismo. Desta forma, este trabalho visa buscar na literatura científica recente responder a seguinte dúvida: Qual a influência dos fatores de riscos modificáveis "sobrepeso" e "sedentarismo" no aparecimento precoce de DM 2 em mulheres com SOP? Tais fatores foram os escolhidos por serem os mais comuns em caso de pré-diabetes. O conhecimento de tais influências e até mesmo se elas atuam de forma diferenciada ou similar são uteis para a orientação a pacientes com SOP com intenção de postergar ao máximo a manifestação da DM 2.

Figure 2 - Word cloud referring to the textual corpus 'health of the field'. Caruaru, Brazil, 2018.

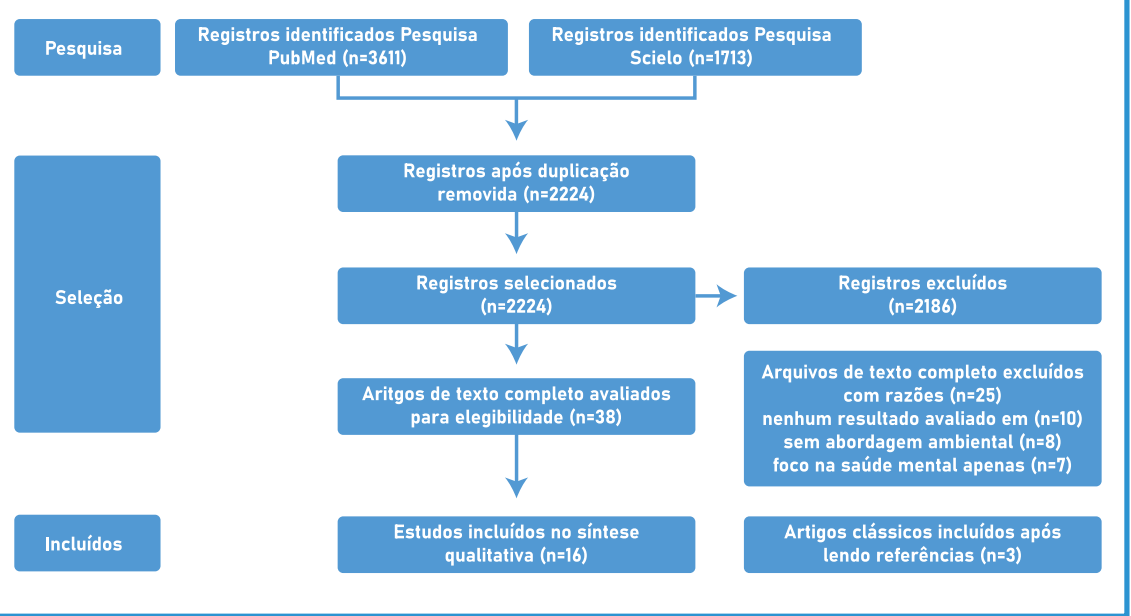

FIGURA 1 - Processo de pesquisa bibliográfica utilizado, contendo as fases de busca e seleção, a fim de encontrar artigos que respondam à questão de pesquisa

1- Estabelecimento dos critérios de inclusăo e exclusăo dos artigos: Eleiçâo de como cs artigos seriam buscados, incluido os criterice para escolha das palavres chaves palavras chaves:

a) Palavras-chaves presentes do DeCS - Descritores em Ciéncias da Saúde,

b) Disponibilidode nos idiomas portugues, ingles e espanhol,

c) Relaçâo direta com o objeto de estudo e com a questăo norteadora dele.

d) Publicados nos útmos 10 anos. Obras raras foram consultadas e consideradas neste trabaho quando a rolováncia da aboeclagem histórica foi significabiva, movida por alguma discussalo entre os artigos encontrados.

e) Năo apresentar conflitos de interesse

II - Pesquisa em sitios oletrōnicos determinados na fase II: Estabelecimento dos criterios ANDIOURNOT para a pesquisa e busca nos sites indexados de maior relevelncia sobre o tema:

a) Pubmed

b) Scielo

IV - Seleçăo dos artigos: Os artigos encontrados na listagem apds inclusd̄o das palavras chaves formam eleilos a partir de tres etapas:

a) Leilura do titulo e avaliaçâo dos mesmos quarto a ligaçâo com a pergunta da pesquisa,

b) Letura do Resumo para me hor compreensao.

c) Letura completa do periodico para seleçâo em prè-avaliaçăo quando em dôvida da sua contribuiçåo para a pesquisa.

V - Colota das informaçōes o confrontamonto com a dúvida da pesquisa: Uso do mopa mental para abordar o problema da pesquișa a luz das indiceçles dos autores dos artigos eleitos.

\section{MÉTODO}

$\mathrm{O}$ processo de pesquisa consistiu em cinco fases, dentro das recomendações PRISMA conforme descrito a seguir na Figura 1.

As palavras chaves eleitas no item nae tapa I, do Item B do método e utilizadas no esquema booleano foram: (síndrome dos ovários policísticos AND diabetes mellitus tipo 2) OR (estilo de vida, obesidade e comportamento sedentário), tendo em vista a questão norteadora da investigação.

\section{RESULTADOS}

Foram encontrados um total de 3611 artigos no PubMed e 1713 no Scielo, no qual 16 atendiam os critérios de seleção da pesquisa visando encontrar proposições que atendesse a dúvida de pesquisa do trabalho, conforme mostra Figura 2:

\section{DISCUSSÃO}

A síndrome dos ovários policísticos (SOP) atinge 4 a $18 \%$ das mulheres em idade reprodutiva no mundo (5). Esta síndrome possui implicações metabólicas tais quais resistência à insulina $(\mathrm{RI})$, tolerância à glicose diminuída, diabetes mellitus tipo 2 e riscos cardiovasculares. Ainda, pode gerar quadros de ansiedade, depressão e uma pior qualidade de vida em pacientes portadoras da síndrome ${ }^{6}$.

A RI resulta no aumento nos níveis plasmáticos deste hormônio, e sua frequência varia em relação a população analisada. Em comparação com a população normal, portadoras da síndrome possuem um valor superior relacionado à RI. No que diz respeito a prevalência por região, mulheres americanas portadoras de SOP com RI variou em torno de $64 \%$, já as italianas atingem em torno de 79,2\%. Considerando o Brasil, a prevalência das portadoras variou de 33 a $70,5 \%{ }^{7}$.

Obesidade e o desenvolvimento de DM 2: fatores ligados a SOP que contribuem para obesidade e DM2

A obesidade atualmente se fixa como 
uma grande preocupação internacional em saúde. Estima-se que o excesso de peso em pacientes com a síndrome possa variar com a idade, raça e fatores ambientais ${ }^{7}$. A obesidade agrava as características hormonais e clínicas da SOP e as mulheres com SOP parecem ter maior risco de obesidade, com vários mecanismos subjacentes que vinculam as condições. A resistência à insulina e o hiperandrogenismo são apontados como o elo entre a síndrome do ovário policístico e a obesidade já que a exposição ao excesso de andrógenos estimula o aumento na expressão de genes relacionados a síntese de ácidos graxos, favorecendo o acúmulo de gordura na cavidade abdominal. Assim, mulheres com SOP ao possuem taxas mais altas de obesidade e adiposidade central em comparação a mulheres sem SOP. Em particular, a gordura visceral desempenha um papel chave na geração do estado resistente à insulina, o que leva a tolerância à glicose diminuída 5 .

A RI mostra-se intrínseca à síndrome, visto que a maioria das mulheres com síndrome do ovário policístico possui essa característica, porém, o sobrepeso e a obe-

\section{FIGURA 7 - Tempo gasto com televisão, celulares e afins: a cada duas horas} têm maior chance de obter obesidade e aumento do risco de DM 2 em até $14 \%$. Entretanto, 1 hora de atividade física intensa reduz em até $22 \%$ o risco.

TEMPO EM FRENTE A COMPUTADOR, CELULAR OU TELEVISÃO E RISCO DE DM

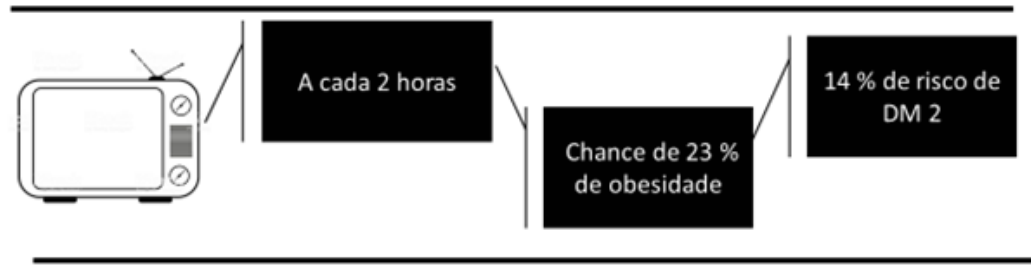

MORAN et al, 2013

TIPO DE EXERCÍCIO- MODERADO E VIGOROSO E REDUÇAÕ DA CHANCE DE DM

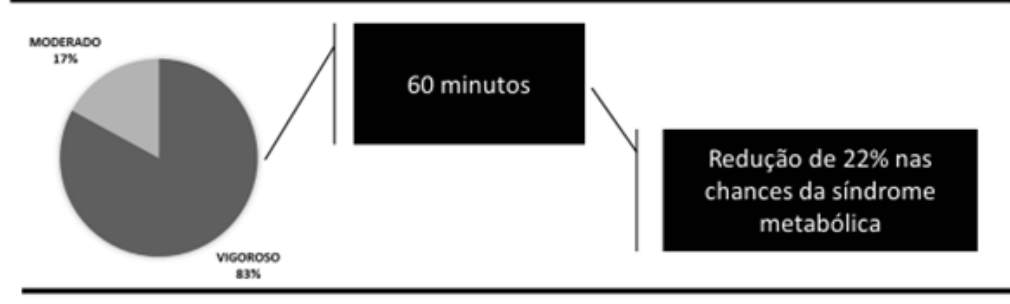

GREENWOOD et al, 2016 obesas e um grupo de não obesas; constatou-se que a prevalência de distúrbios metabólicos se fez elevada em ambos os grupos evidenciando que a presença da síndrome favorece o desenvolvimento de comorbidade independente do IMC. Entretanto mulheres obesas mostraram uma prevalência de três alterações metabólicas: resistência à insulina, intolerância à glicose diminuída e síndrome metabólica evidenciando um maior risco de desenvolvimento da diabetes mellitus tipo II. Assim, ao correlacionar a RI de acordo com o IMC, observou-se que a frequência de RI aumente progressivamente com o IMC 9.

Embora a maioria das publicações mostre que o excesso de peso pode exacerbar alguns distúrbios metabólicos, alguns estudos sugerem que a obesidade tem um discreto impacto não demonstrando tamanho impacto na síndrome ${ }^{5}$. A sensibilidade e a resistência à insulina, decorrente de um defeito intrínseco do receptor insulínico no qual ocorre a fosforilação da serina mostra-se presente, independente do peso corporal da mulher. Entretanto, o tecido adiposo, órgão endócrino capaz de secretar diversas substâncias que interferem no metabolismo dos carboidratos e lipídios, quando em excesso, acaba por agravar a resistência à insulina. Dessa forma, a obesidade e a distribuição de gordura abdominal apresentam um papel importante na manutenção da SOP, podendo agravar as manifestações da síndrome e aumentar o risco para com a formação a diabetes mellitus $2^{10}$.

Sedentarismo e diabetes mellitus tipo 2: avaliação dos riscos de desenvolvimento da DM2

Mulheres com SOP podem ter um estilo de vida mais desfavorável quando em comparação com mulheres saudáveis. Quando analisado o estilo de vida, mudanças devem ser feitas, principalmente em mulheres que se encontram obesas, em prol do bem-estar para a paciente ${ }^{11}$.

A combinação de exercícios aeróbicos intercalado com exercícios de alta intensidade realizado semanalmente em torno de 150 a 175 min contribui contra a RI, 


\section{artigo}

Rosin, B.E.; Machado, F.R.A.; Rosa, S.M.; Paszcuk, A.M.; Karstens, L.F.; Pinto, L.H.

Influência dos fatores "sobrepeso" e "sedentarismo" no aparecimento precoce de diabetes em mulheres com SOP

tolerância a glicose diminuída e diabetes mellitus tipo $2^{12}$. No entanto, estudos afirmam que a incorporação da atividade física deve ser moderada e simples, de 30 minutos ao dia, no qual a meta deveria ser o benefício geral da saúde e não a perda do peso em $\mathrm{si}^{6}$.

A cada 60 minutos gastos na atividade observa-se uma redução de $22 \%$ nas chances da síndrome metabólica 13 conforme mostra a Figura 7:

\section{CONCLUSÃO}

Considerando que a questão central deste trabalho estava relacionada a influência dos fatores de riscos modificáveis "sobrepeso" e "sedentarismo" no apare- cimento precoce de DM 2 em mulheres com SOP, mesmo que não sejam diagnosticadas como diabéticas, o fato de serem mulheres em situação de risco para esta condição clínica ou serem já pré-diabéticas requer uma atenção especial.

Os custos atribuídos ao cuidado com diabéticos e pré-diabéticos são elevados no mundo todo. Nos Estados Unidos da América, tem-se atualmente cerca de 21 milhões de adultos abaixo de 30 anos com diabetes ${ }^{15,16}$.

Em um breve levantamento em Planos Municipais de Saúde de capitais da região Sul do Brasil, não se encontrou nenhuma ação específica para cuidados de mulheres com SOP. Considerando proporção de 3,26 de chance de desen- volverem a TGD, RI e DM2 em relação a mulheres que não possuem $S O P$, é que se traz a questão da necessidade de se ter uma ação mais específica para as mulheres portadoras de tal síndrome afim de evitar a manifestação precoce de DM 2. Isto inclui estimulo e promoção a atividades físicas e rastreamento mais efetivo de DM 2 em idades mais jovens nesse grupo de mulheres.

\section{AGRADECIMENTOS}

Os pesquisadores agradecem a Univille e ao Fundo de Apoio à Pesquisa que possibilita o desenvolvimento do Projeto Integrado Impactos Ambientais e SaúdeECOSAM.

\section{REFERÊNCIAS}

1. Do Carmo Silva R, Pardini DP, Kater CE. Síndrome dos ovários policísticos, síndrome metabólica, risco cardiovascular e o papel dos agentes sensibilizadores da insulina. Vol. 50, Arquivos Brasileiros de Endocrinologia e Metabologia. 2006. p. 281-90.

2. Antonio J, Marcondes M, Roberto C, Barcellos G, Rocha MP. Dificuldades e armadilhas no diagnóstico da síndrome dos ovários policísticos Difficulties and pitfalls in the diagnosis of polycystic ovary syndrome. Vol. 55, Arq Bras Endocrinol Metab. 2011.

3. Anagnostis P, Tarlatzis BC, Kauffman RP. Polycystic ovarian syndrome (PCOS): Long-term metabolic consequences. Metabolism. 2018 Sep 1;86:33-43.

4. de Moura HHG, Costa DLM, Bagatin E, Sodré CT, Manela-Azulay M. Síndrome do ovário policístico: Abordagem dermatológica. Vol. 86, Anais Brasileiros de Dermatologia. 2011. p. 111-9.

5. Leão LM. Obesidade e síndrome dos ovários policísticos: vínculo fisiopatológico e impacto no fenótipo das pacientes. Rev Hosp Univ Pedro Ernesto. 2014 Mar 17;13(1).

6. Teede H, Deeks A, Moran L. Polycystic ovary syndrome: a complex condition with psychological, reproductive and metabolic manifestations that impacts on health across the lifespan. BMC Med [Internet]. 2010 Dec 30 [cited 2019 Dec 3];8(1):41. Available from: http://bmcmedicine.biomedcentral.com/articles/10.1186/1741-7015-8-41

7. Lamarca A, Orvieto R, Giulini S, et al. Mllerian-inhibiting substance in women with polycystic ovary syndrome: Relationship with hormonal and metabolic characteristics. Fertility and Sterility 2004; 82: 970-972.

8. Kakoly NS, Khomami MB, Joham AE, Cooray SD, Misso ML, Norman RJ, et al. Ethnicity, obesity and the prevalence of impaired glucose tolerance and type 2 diabetes in PCOS: A systematic review and meta-regression. Hum Reprod Update. 2018
Jul 1;24(4):455-67.

9. Melo AS, Macedo CSV, Ferriani RA, Navarro PA de AS, Romano LGM. Women with polycystric ovary syndrome have a higher frequency of metabolic syndrome regardless of body mass index. Rev Bras Ginecol e Obstet. 2012;34(1):4-10.

10. Romano LGM, Bedoschi G, Melo AS, de Albuquerque FO, Rosa e Silva ACJ de S, Ferriani RA, et al. Anormalidades metabólicas em mulheres com síndrome dos ovários policísticos: Obesas e não obesas. Rev Bras Ginecol e Obstet. 2011 Jun;33(6):310-6.

11. Moran LJ, Ranasinha S, Zoungas S, McNaughton SA, Brown WJ, Teede HJ. The contribution of diet, physical activity and sedentary behaviour to body mass index in women with and without polycystic ovary syndrome. Hum Reprod. 2013;28(8):2276-83.

12. Anwar S, Shikalgar N. Prevention of type 2 diabetes mellitus in polycystic ovary syndrome: A review. Vol. 11, Diabetes and Metabolic Syndrome: Clinical Research and Reviews. Elsevier Ltd; 2017. p. S913-7.

13. Greenwood EA, Noel MW, Kao CN, Shinkai K, Pasch LA, Cedars $\mathrm{Ml}$, et al. Vigorous exercise is associated with superior metabolic profiles in polycystic ovary syndrome independent of total exercise expenditure. Fertil Steril. 2016 Feb 1;105(2):486-93.

14. Brennan L, Teede H, Skouteris H, Linardon J, Hill B, Moran L. Lifestyle and Behavioral Management of Polycystic Ovary Syndrome. J Women's Heal. 2017 Aug 1;26(8):836-48.

15. Mcevoy JW, Windham BG, Ballantyne CM, Selvin E. Mortality Implications of Prediabetes and Diabetes in Older Adults. 2019;1-7.

16. Schneider ALC, Kalyani RR, Golden S, Stearns SC, Wruck L, Yeh $\mathrm{HC}$, et al. Diabetes and prediabetes and risk of hospitalization: The atherosclerosis risk in communities (ARIC) study. Diabetes Care. 2016;39(5):772-9. 